\title{
Electrical and Thermal Characterization of Electrospun PVP Nanocomposite Fibers
}

\author{
Waseem S. Khan, ${ }^{1}$ Ramazan Asmatulu, ${ }^{1}$ and Mohamed M. Eltabey ${ }^{2,3}$ \\ ${ }^{1}$ Department of Mechanical Engineering, Wichita State University, 1845 Fairmount, Wichita, KS 67260-0133, USA \\ ${ }^{2}$ Basic Engineering Science Department, Faculty of Engineering, Menoufiya University, Shebin El-Kom, Egypt \\ ${ }^{3}$ Medical Physics Department, Faculty of Medicine, Jazan University, Saudi Arabia
}

Correspondence should be addressed to Ramazan Asmatulu; ramazan.asmatulu@wichita.edu

Received 30 October 2012; Revised 30 January 2013; Accepted 6 February 2013

Academic Editor: Theodorian Borca-Tasciuc

Copyright (C) 2013 Waseem S. Khan et al. This is an open access article distributed under the Creative Commons Attribution License, which permits unrestricted use, distribution, and reproduction in any medium, provided the original work is properly cited.

Polyvinylpyrrolidone (PVP) solutions incorporated with multiwall carbon nanotubes (MWCNTs) were electrospun at various weight percentages, and then the electrical resistance and some thermal properties of these nanocomposite fibers were determined using a high-accuracy electrical resistance measurement device. During the electrospinning process, system and process parameters, such as concentrations, applied voltage, tip-to-collector distance, and pump speeds, were optimized to receive the consistent nanocomposite fibers. When polymers are used in many industrial applications, they require high electrical and thermal conductivities. Most polymers exhibit low electrical conductivity values; however, in the presence of conductive inclusions, the electrical resistance of the MWCNT fibers was reduced from $50 \mathrm{M} \Omega$ to below $5 \mathrm{M} \Omega$, which may be attributed to the higher electrical conductivities of these nanoscale inclusions and fewer voids under the applied loads. This study may open up new possibilities in the field for developing electrically conductive novel nanomaterials and devices for various scientific and technological applications.

\section{Introduction}

1.1. General Background. Electrospinning is a versatile method to produce ultrafine polymeric fibers. It has attracted enormous attention due to the numerous options for fabricating ultrafine fibers [1]. When the diameters of polymer are reduced to the nanoscale, some exotic characteristics appear, such as high surface area-to-volume ratios, mechanical flexibility, and superior mechanical properties, compared to their bulk-size counterparts [2]. During electrospinning, a high voltage and consequently high electrostatic field are applied to a polymer solution at the end of a capillary tube. The electrostatic field introduces charges in the solution when the mutual repulsions between charges reach a threshold value, and a fluid jet forms a conical droplet at the tip of a capillary tube, resulting in the formation of a Taylor cone $[3,4]$. The jet travels in a straight path, called the jet's length, experiences bending instabilities to form spiral motions, and finally is collected on a screen, which is either grounded or connected to a DC supply of opposite polarity. Figure 1 shows a general schematic view of the electrospinning process.

Parameters such as load, temperature, and inclusion have a direct relationship to the electrical and thermal resistances of the modified fibers. When the load is applied on a film of the fibers, individual fibers and molecules of polymer move closer together where the heat conduction becomes easier, and electrical resistance is reduced drastically due to the lower separation distances, higher number of charge carrier/holes (free mobile particles carrying electrical charges), and polarizability. The increase in mobility of the charge carriers with temperature is governed by the following Arrhenius dependent equation $[5,6]$ :

$$
\mu_{t}=\mu_{o} e^{\left(-E_{\mu} / R T\right)}
$$

where $\mu_{t}$ is the mobility at temperature $T\left({ }^{\circ} \mathrm{K}\right), E_{\mu}$ is the activation energy, $R$ is Boltzmann's constant, and $\mu_{0}$ is a 


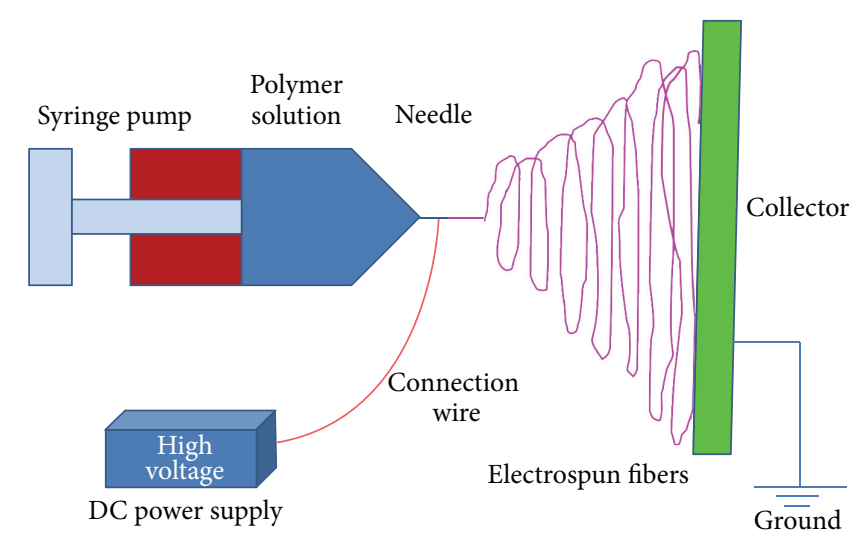

FIGURE 1: Schematic view of an electrospinning process.

temperature-independent constant. The basic expression for the electrical conductivity, $K_{p}$, is [7]

$$
K_{\rho}=\sum n_{i} e_{i} \mu_{i}
$$

where $n_{i}$ is the density of the charge carriers, and $e_{i}$ and $\mu_{i}$ are the corresponding charges and mobility of the charge carriers, respectively. The factors that mainly influence the electrical resistance of nanocomposite fibers under load and temperature are related to the hysteresis effects, charge carrier concentration, polarizability, and porosity/void. When multiwall carbon nanotubes (MWCNTs) are added into polymers, the charge carrier concentration increases, as does the conduction rate. At higher temperatures, the mobility of the charge carriers increases and, likewise, the conduction. Increasing the wt $\%$ of MWCNTs increases the dipole moments in the polymer, which in turn enhances the polarizability of the materials and thereby conduction. Porosity is the amount of voids or empty spaces presented in a material. This is an important physical property that affects almost all physical properties of the material. The dynamic lag between input and output is commonly referred to as hysteresis, or rate-dependent hysteresis. This effect is generally observed during the unloading of a material after applying tensile stresses. This phenomenon is common in ductile materials, such as rubber and plastic. The percolation behavior of an electrical conduction in nanotubes-polymer composites in a bulk and thin film has been extensively studied for many years [8]. The high aspect ratio of CNTs is beneficial in making percolation network at lower loading of the inclusions.

There are some other studies on the nanotubes-based polymeric electrospun nanocomposite fibers for various purposes [9-13]. A detailed study of the electrical and thermal properties of electrospun fibers was presented in our study. Thermal analysis is an important analytical technique for understanding the structural/property relationship of a polymeric material, and it is also a helpful method to determine the thermal stability of the materials [9]. Differential scanning calorimetry is a technique to characterize the thermal stability of a polymer. DCS determines the heat flow rate associated with thermal changes that can be measured as a function of temperature and time. DSC technique can also be used to study the melting behavior and phase transition of the materials. The objective of this work was to study the electrical transport properties and thermal stability of nanotubes-based polymeric fibers. The study of variation of the temperature dependent electrical conduction of nanocomposite fibers can provide an insight into the mechanism of charge and heath transport [8].

The nanophase/nanoize materials that serve as fillers in the polymeric matrices are the clusters of atoms or molecules having size dependent properties, which are different from their bulk counterparts [10]. Polymer nanocomposites are of great importance due to their novel properties resulting from the excellent combination of parents constituents into a single phase [11]. Polymer nanocomposites have been finding extensive applications owing to their easy processing, low manufacturing cost, and unique physicochemical properties [12]. Electrospinning is a rapid, easy and low cost process for many industrial applications [13].

1.2. Polymer Nanocomposites. Polymer-carbon nanotube (CNT) composites have recently attracted a considerable amount of scientific interest because of their structural characteristics, such as high aspect ratio, surface area, and electrical, thermal, and mechanical properties, all of which are positive signs for the fabrication of ultralightweight and robust composites [14]. The biggest challenge in fabricating such composites is the uniform dispersion of CNTs in a polymer matrix, since van der Waals forces between individual nanotubes cause agglomeration, which reduces the expected properties of nanocomposites [14]. A good dispersion technique is essential for allowing the formation of an interconnecting filler network of CNTs at a lower concentration without changing the electrical resistivity. CNTs can provide electrical conduction at lower concentrations in the nanocomposite structure. This property is required in commercial applications to dissipate electrostatic charge and thermal heat [14]. In the present study, in order for CNTs to retain their properties, CNTs were physically dispersed in a polymer matrix instead of grafting. 


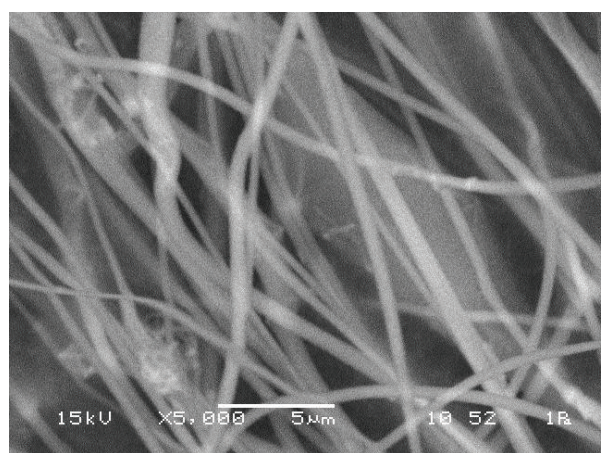

(a)

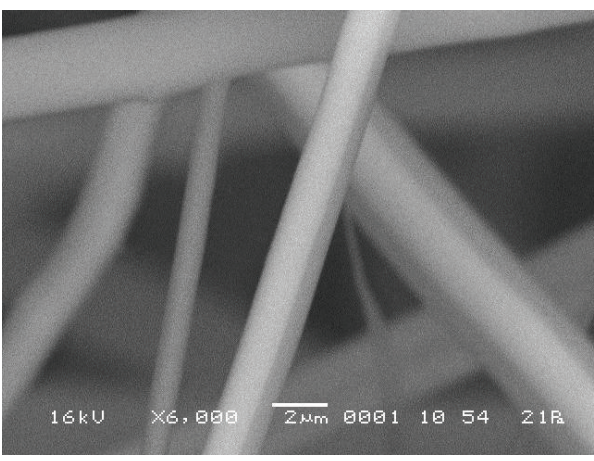

(c)

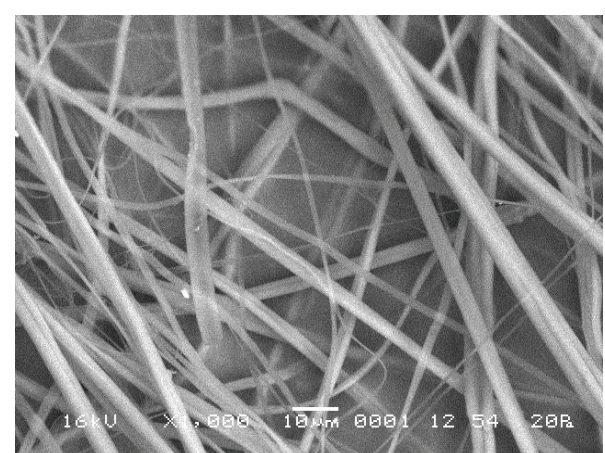

(b)

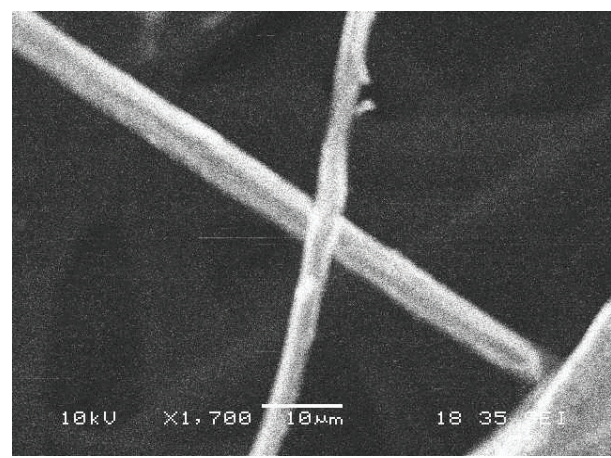

(d)

FIGURE 2: SEM images of PVP nanocomposite fibers incorporated with the following wt\% MWCNTs: (a) 0, (b) 0.5 , (c) 1 , and (d) 2. Electrospinning experiments conducted at $85: 15 \mathrm{PVP}$ and ethanol ratio, $25 \mathrm{kV}$ DC voltage, $20 \mathrm{~cm}$ separation distance, and $2 \mathrm{~mL} / \mathrm{hr}$ pump speed.

\section{Experimental}

2.1. Materials. Polyvinylpyrrolidone (PVP) with a molecular weight of $130,000 \mathrm{~g} / \mathrm{mole}$ was purchased from the SigmaAldrich company. Multiwall carbon nanotubes with a diameter of $140 \pm 30 \mathrm{~nm}$ and a length of $7 \pm 2$ microns were purchased from the Fisher Scientific company. Different wt $\%$ $(0,1,2$, and 4$)$ of MWCNTs was mechanically dispersed in ethanol, and PVP polymer in granular form was added to the dispersion prior to the 30 -minute sonication. The dispersion was constantly stirred at $60^{\circ} \mathrm{C}$ for 12 hours before the electrospinning process. Special care was taken to disperse the MWCNTs uniformly; otherwise, the electrospinning process and/or nanocomposite fiber morphology would deteriorate.

2.2. Methods. The prepared dispersion was transferred to a $10 \mathrm{~mL}$ plastic syringe connected to a capillary needle with an inside diameter of $0.3 \mathrm{~mm}$. A platinum electrode inside the syringe was attached to a high DC voltage supply. In this study, the applied voltage (18-20 kV), the syringe pump speed (1-3 $\mathrm{mL} / \mathrm{hr})$, and the distance between capillary tube and grounded counter electrode $(15-25 \mathrm{~cm})$ were tested to optimize the electrospinning parameters. The grounded counter electrode was a flat aluminum foil placed at a distance of 15$25 \mathrm{~cm}$ from the capillary tube. Electrospun fibers were then collected on a grounded screen and dried in an oven at $60^{\circ} \mathrm{C}$ for 6 to 8 hours to remove all residual solvents. A scanning electron microscope (SEM) (JEOL Model JSM-6460LV) was used to identify the morphology and surface characteristics of the nanocomposite fibers. Figure 2 shows the selected SEM images of the PVP electrospun nanocomposite fibers. A commercial differential scanning calorimeter (DSC) (model Q1000) was used to measure the glass transition temperature $\left(T_{g}\right)$, heat flow, and heat flow derivative of the specimens. Dry nitrogen gas was purged through the DSC cell during the experiments, each of which had $10 \mathrm{mg}$ of samples in an aluminum hermetic pan. During the tests, samples were heated from $0^{\circ} \mathrm{C}$ to $300^{\circ} \mathrm{C}$ at the rate of $20^{\circ} \mathrm{C} / \mathrm{min}$.

A homemade electrical resistance device was built, tested on the known thin films, and then used for the electrical characterizations of the electrospun nanocomposite fibers [4]. A thin film of the fibers was positioned between two cylinders $(2.5 \mathrm{~cm}$ in diameter $)$ of a hydraulic press on which two probes of a highly sensitive multimeter were placed to record the electrical resistance values of the samples. A thin coating of a conductive paste was applied on cylinders in order to avoid contact resistance. The applied loads were varied from 0 to 9 tons $\left(0-1440 \mathrm{~kg} / \mathrm{cm}^{2}\right)$ during the resistance measurements. Each sample was externally loaded 3-5 times and the resistance changes recorded.

\section{Results and Discussion}

3.1. Loads versus Electrical Resistance of PVP Fibers. In each test, the same nanocomposite fiber films were externally loaded five times during the measurements. Figure 3 shows 


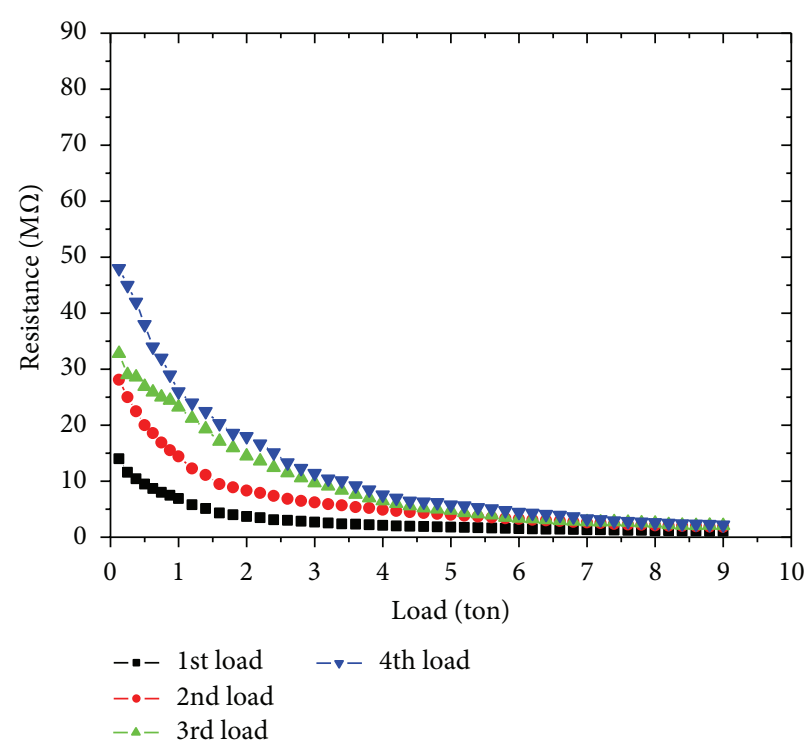

(a)

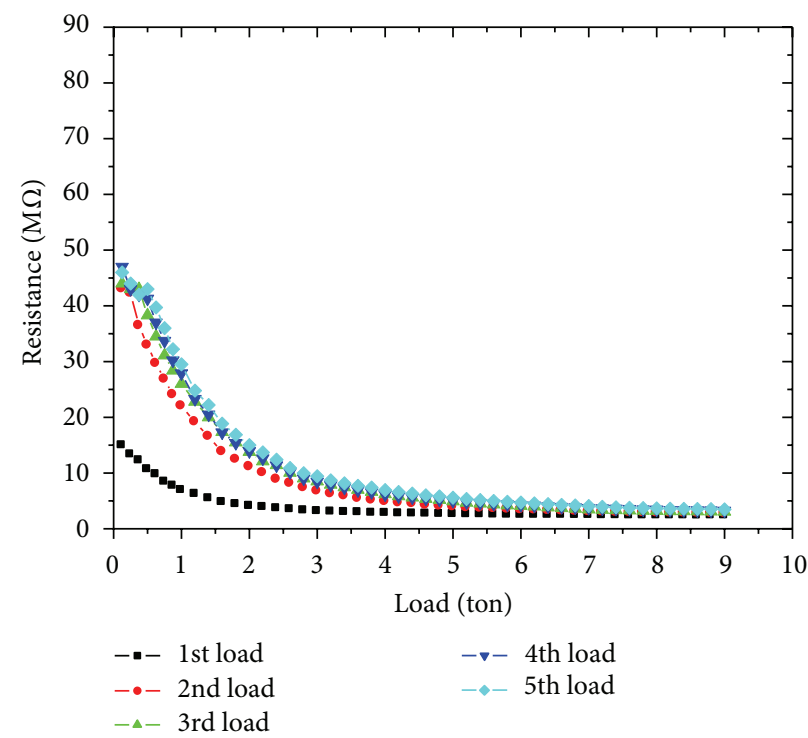

(c)

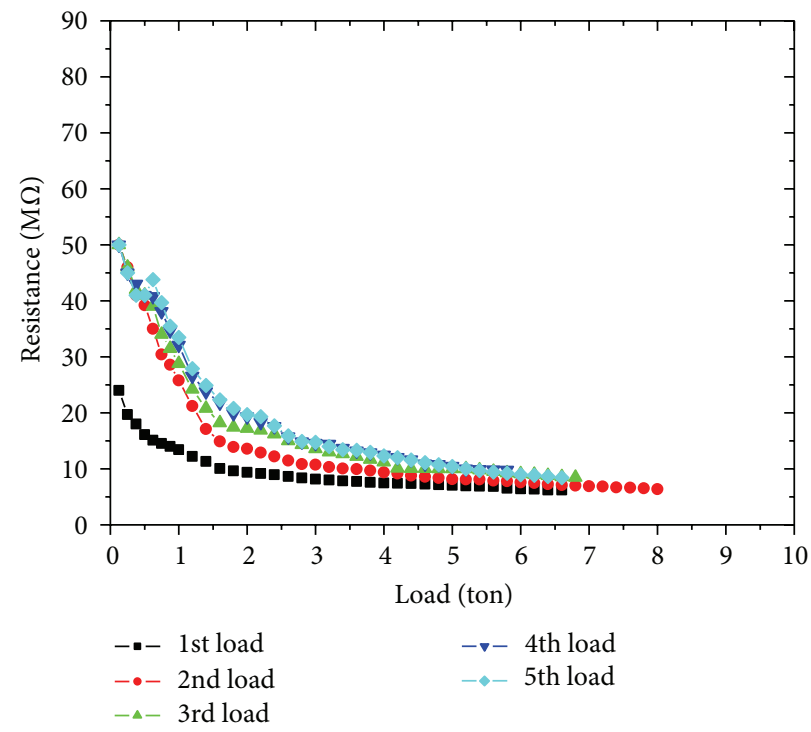

(b)

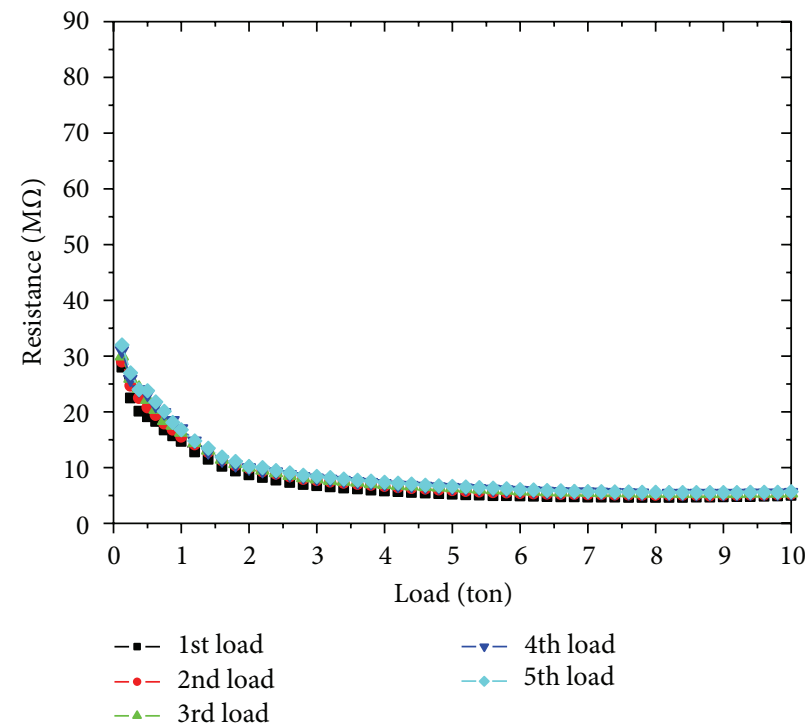

(d)

Figure 3: Load versus electrical resistance curves of PVP nanocomposite fibers obtained at following wt $\%$ MWCNTs: (a) 0 , (b) 1 , (c) 2, and (d) 4 .

the load versus electrical resistance values of the PVP nanocomposite fibers at various percentages of MWCNTs. As expected, resistance decreases with the applied load for all tested samples. This behavior may be explained using the following equation [15]:

$$
R_{\text {resistance }}=\rho\left(\frac{L}{A}\right),
$$

where $\rho, L$, and $A$ are resistivity, thickness, and crosssectional area of the sample, respectively. When the load is increased on nanocomposite fibers, two major changes in thickness and porosity can be observed on the samples. The thickness of the sample decreases under the load, and the contact area between the fibers and electrode increases, which in turn reduces the overall electrical resistance. At $0 \mathrm{wt} \%$ of MWCNTs (Figure 3(a)), the resistance increases in each trial when the load is removed, which may be attributed to the hysteresis and porosity changes, whereas at 1 and $2 \mathrm{wt} \%$ MWCNTs (Figures 3(b) and 3(c)), the porosity of the fiber film is likely to be reduced because of the conductive nanoscale inclusions. Resistance increases only in the first trial after unloading, and in subsequent trials, the resistance curves overlapped due to the higher wt $\%$ of MWCNTs, which is expected to reduce the porosity and electrical resistance of nanocomposite fibers significantly.

At a higher loading of MWCNTs, the resistance curves (Figure 3(d)) overlap each other, which may be due to the fact that at high loading, polarizability and charge carrier 


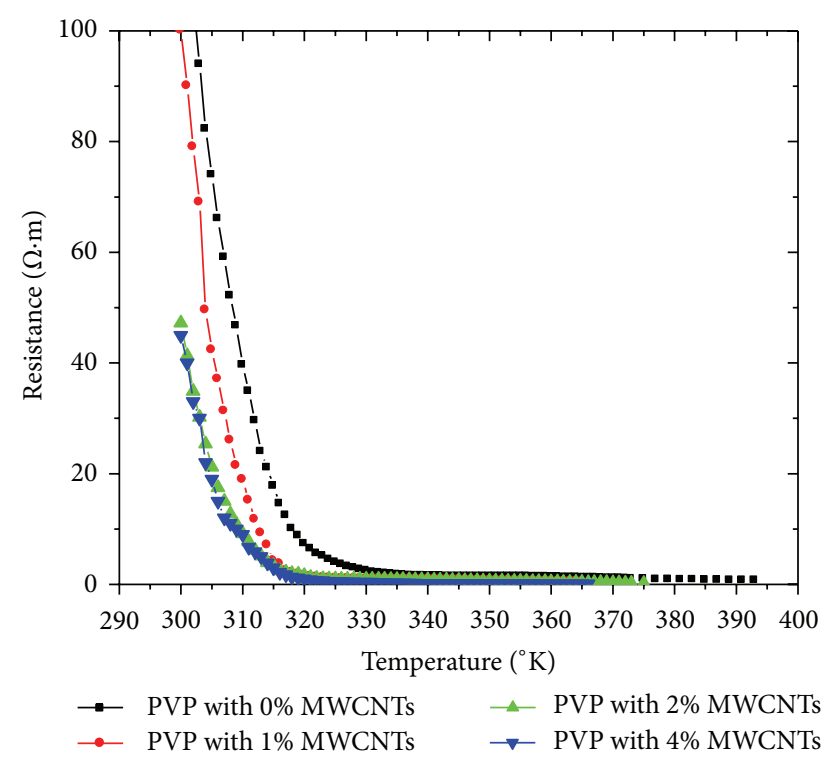

Figure 4: Temperature versus electrical resistance curves of PVP nanocomposite fibers obtained at $0,1,2$, and $4 \mathrm{wt} \%$ MWCNTs.

concentrations of the nanocomposite fibers are also increased. Adding MWCNTs into the polymeric fibers increases both the electrical and thermal conductivities of the fibers [4]. At higher MWCNTs, there is no shift between successive trials, since MWCNTs make a nanocomposite conductive enough to reduce the electrical resistance. At lower MWCNT contents, possibly the proper percolating network structure was not established, and therefore, the resistivity remained high [16]. However, when the wt.\% of MWCNTs reached at $4 \%$, a continuous nanotube network was established to significantly drop the electrical resistance. For example, the resistance of pure PVP fiber was $50 \mathrm{M} \Omega$, which was reduced to $30 \mathrm{M} \Omega$ at $4 \mathrm{wt} . \%$ of MWCNTs without any loading. Once the network of MWCNTs is established, a pathway is created to support the electron transportation process among carbon nanotubes [17]. Zhu et al. [17] established a relationship between the aspect ratio $(L / r)$, where $L$ and $r$ are the length and radius of an individual nanofiber and percolation threshold (Pc). The aspect ratio of MWCNTs used in this study is 100 . The percolation threshold was established at $4 \mathrm{wt} \%$ of MWCNTs, indicating a high aspect ratio of the nanocomposite fibers.

\subsection{Temperature versus Electrical Resistance of PVP Fibers.}

Figure 4 shows the effects of temperatures on the electrical resistance of nanocomposite fibers at a constant loading. A K-Type thermocouple was attached on the top surface of the fiber for recording temperature readings, while two probes of a multimeter were attached on either side of the film of the fibers to record electrical resistance. The whole assembly was enclosed in glass fiber insulation in order to avoid thermal losses. As shown in Figure 4, increasing the sample temperature drastically reduced the electrical resistance. The relation between temperature and resistivity may be governed by the following Arrhenius dependent equation $[6,7,14,15$, 18]:

$$
\rho=\rho_{0} e^{\left(E_{\mu} / R T\right)},
$$

where $\rho$ is the resistivity at temperature $T(K), E_{\mu}$ is the conduction activation energy, $R$ is the Boltzmann's constant, and $\rho_{0}$ is the temperature-independent constant. As the temperature increases, the molecules undergo a phase change (e.g., rubbery and partly solid) and likely slide over each other to facilitate the electrical conductions. This drastically decreases the overall electrical resistances of the fibers (Figure 4).

There is a close correlation among the conductivity, aspect ratio, concentration, and dispersion of CNTs filler materials, all of which influence the particle-particle and particlematrix interactions [19]. The existence of conductivity can be attributed to the formation of conductive pathways, when the wt $\%$ of MWCNTs exceeds a critical proportion, called the percolation threshold. At a low percentage of MWCNTs, the distance between particles (MWCNTs) is large, resulting in a lower conductivity. The percolation threshold exists at higher nanoscale inclusions, and in the absence of a conductive pathway, the polymeric materials are mostly called insulators $[16,19,20]$. At a high percentage of MWCNTs, the MWCNTs tend to agglomerate due to the lack of the dispersion and interfacial interactions, and subsequently impede the formation of conductive pathways [19]. Therefore, a good dispersion is necessary to establish the conductive pathway in the nanocomposite fibers.

Phonon conduction by a percolated network of MWCNTs leads to an increase in thermal and electrical conductivity by several order of magnitudes when the required filler content is reached to establish percolation threshold [20]. The percolation threshold decreases with increasing aspect ratio of filler material present in the polymer matrix [21]. CNTs provide electrical conduction when incorporated into the polymer matrix. Test results showed that a small concentration of CNTs would be needed to obtain relatively high electrical conductions, which is useful in a wide range of industrial applications [22]. Electron transport in composites usually involves thermally activated transport through the network of charge carriers between nearest-neighbor particles at close proximity forming an overlap of electron wave function of each particle [21]. The formation of a MWCNT conduction pathway allows the electrons to travel through the composite structure of the fibers. The MWCNTs consist of concentric multiwalled tubes with one end capped; therefore, only the outer layer contributes to the electron transfer that gives the nanocomposites their electrical properties [23].

Since the mechanism of heating the polymer above room temperature is thermally induced, the resistance versus temperature behavior may be understood by the activation energy values of the nanocomposite fibers. Figure 5 shows the plot of the $\log R$ and $1000 / T$ at $0,1,2$, and $4 \mathrm{wt} \%$ of MWNTs. This plot shows a linear behavior and can be divided into three distinct zones, indicated by green, blue, and red lines. The second zone shows the exponential function, which clearly indicates that the thermal process follows the 


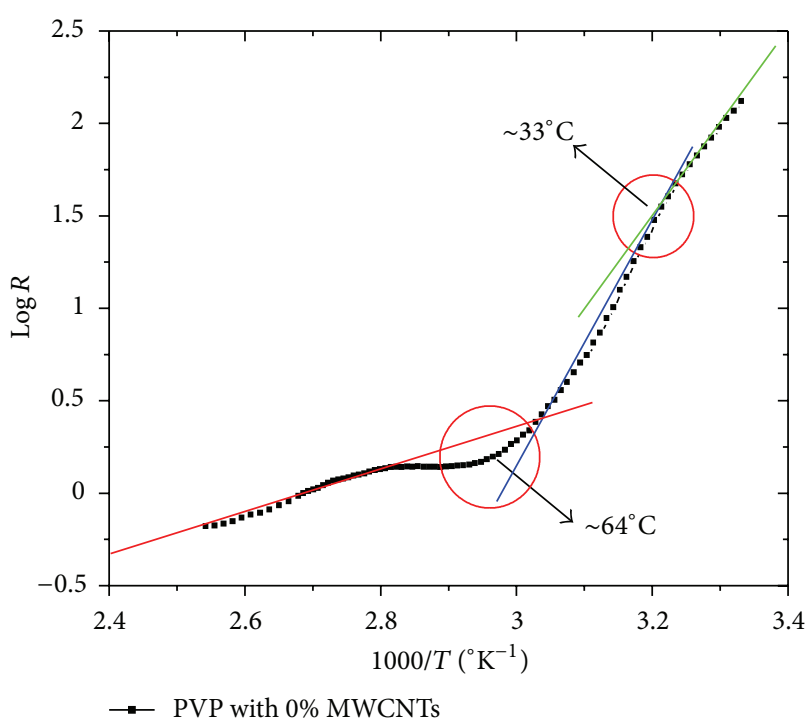

(a)

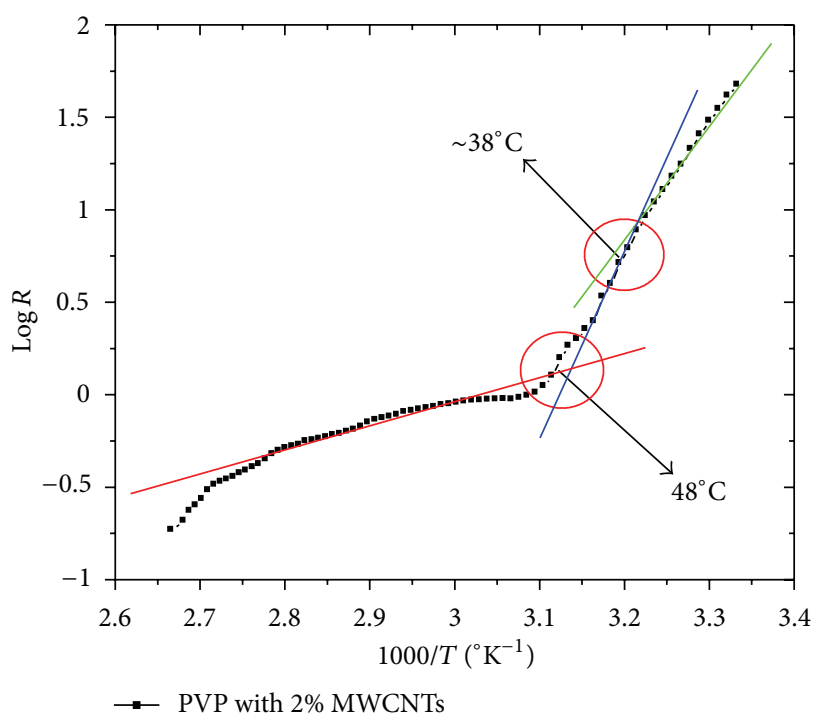

(c)

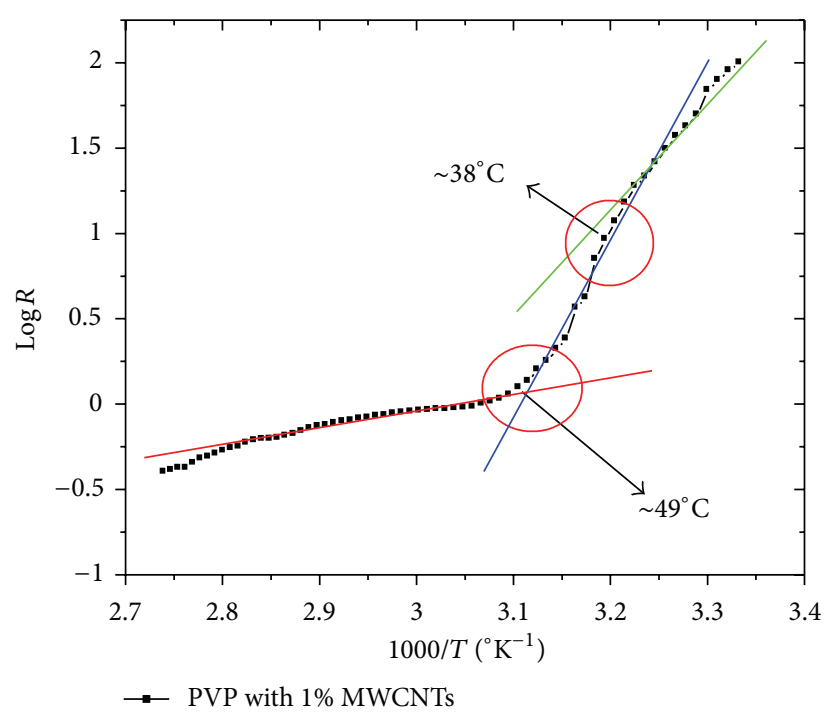

(b)

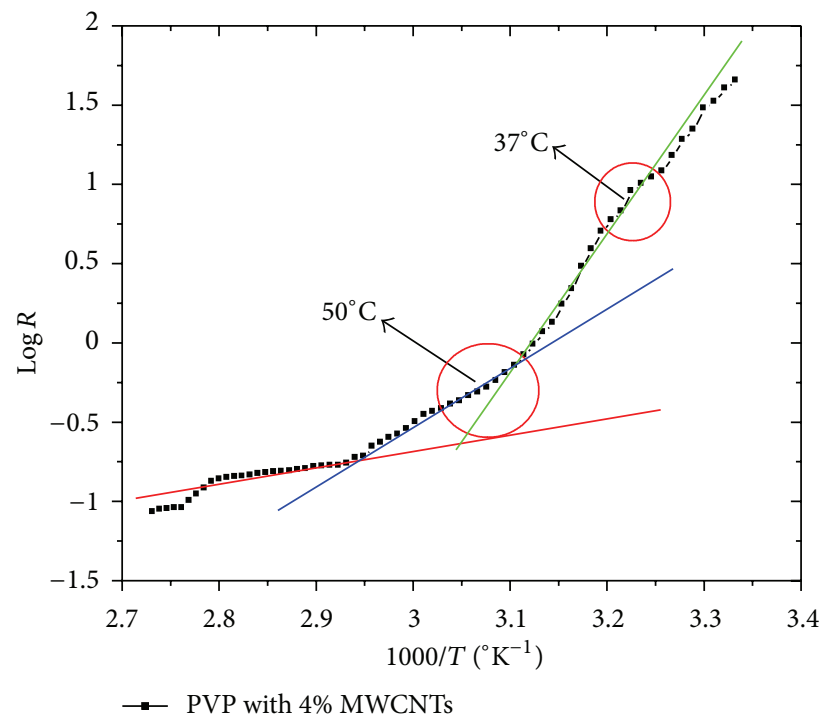

(d)

Figure 5: $\log R$ versus 1000/T curves of PVP nanocomposite fibers obtained at various wt\% MWCNT inclusions: (a) 0, (b) 1, (c) 2, and (d) $4 \mathrm{wt} \%$.

Arrhenius equation. The activation energy of the electrospun $\mathrm{PVP} / \mathrm{MWCNT}$ composite is influenced by the reinforcement effect of nanotubes, increased cross-linking density, and restricted mobility of the polymer and nanotube interactions.

Figure 6 is the $\log R$ versus $1 / T$ plot for 0,2 , and $4 \mathrm{wt} \%$ of MWCNTs in PVP polymer. This plot shows a linear relationship between the $\log R$ and $1 / T$, indicating a good fit for the Arrhenius equation. From Figure 6, the activation energy values were calculated as $48.37 \mathrm{KJ} / \mathrm{mole}(0.50 \mathrm{eV})$, $62.88 \mathrm{KJ} / \mathrm{mole}(0.65 \mathrm{eV})$, and $76.96 \mathrm{KJ} / \mathrm{mole}(0.79 \mathrm{eV})$ for 0 , 2 , and $4 \mathrm{wt} \%$ of MWCNTs in PVP nanocomposite fibers, respectively. Results also indicate that the mobility of the PVP chain in an amorphous region was restricted by the presence of the nanotubes [24-28]. That may be the possible reason for why activation energies were increased as the wt $\%$ of highmodulus MWCNTs increased. When the polymer is strained, it must overcome the energy barrier in order to allow the polymer chains to move around and rotate the chain bonds. Thus, this energy barrier may be the activation energy of the polymeric system [29].

Figure 7 shows the heat flow and heat flow derivativevalues. The derivative of the heat flow shows a phase transition, which is a second order and takes place in the temperature range of $37^{\circ} \mathrm{C}$ to $60^{\circ} \mathrm{C}$. In the presence of MWCNTs, the heat flow decreases as the wt $\%$ of MWCNTs increases because of the inclusion properties (Figure 7). The thermal conductivity of MWCNTs used in this study is $1600 \mathrm{w} / \mathrm{m}^{\circ} \mathrm{K}$, which is 3 to 4 times higher than most common metals. The addition of 


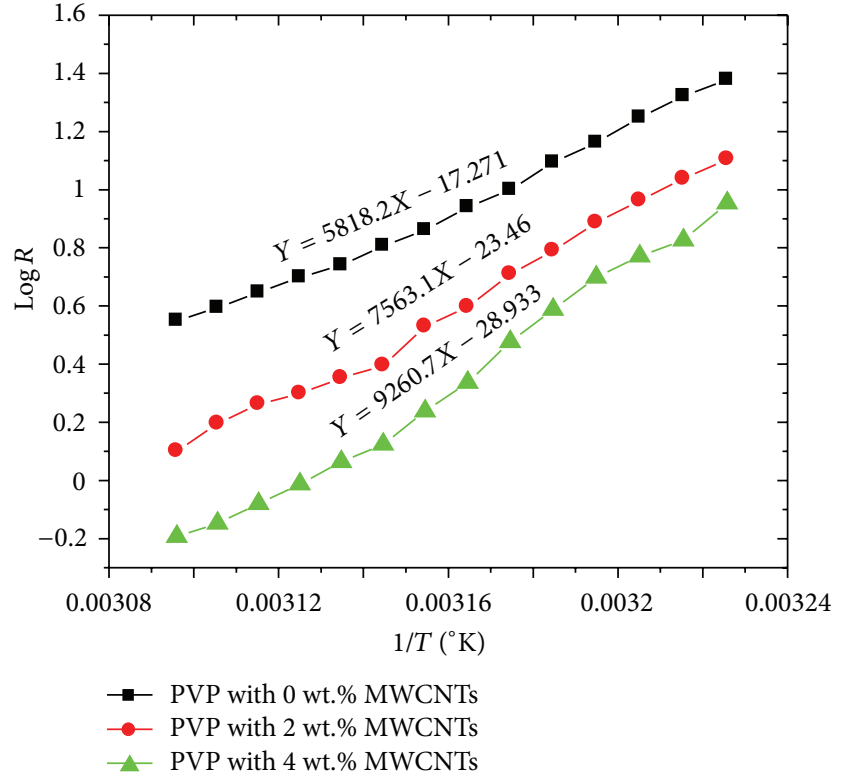

Figure 6: $\log R$ versus $1 / T$ curves of PVP nanocomposite fibers obtained at $0 \mathrm{wt} \%, 2$, wt $\%$, and $4 \mathrm{wt} \%$ MWCNT inclusions.

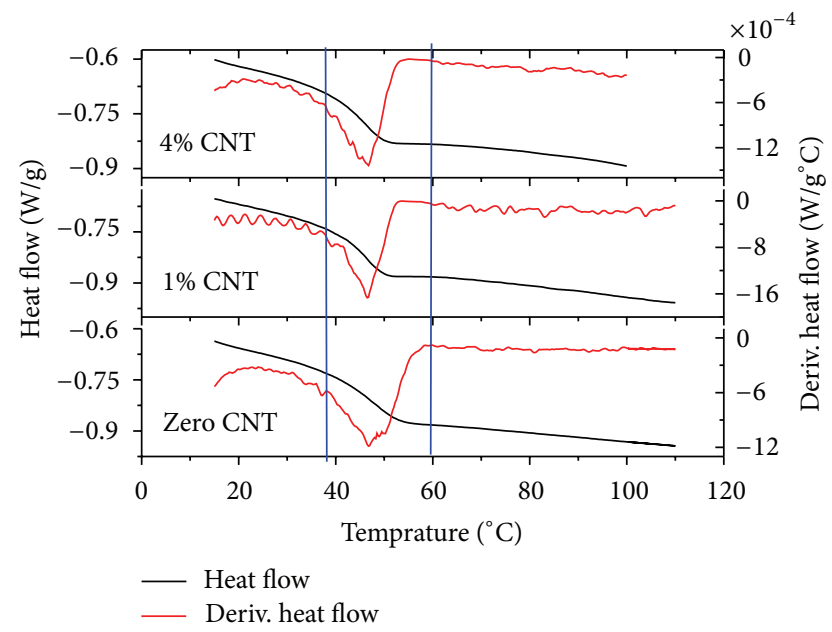

Figure 7: Heat flow and heat flow derivative of nanocomposite fibers incorporated with $0 \mathrm{wt} \%, 1 \mathrm{wt} \%$, and $4 \mathrm{wt} \%$ MWCNTs.

MWCNTs makes the conduction process easier, owing to the increased polarizability values of the nanocomposite fibers.

\subsection{DSC Analysis of PVP Nanocomposite Fibers. Figure 8} shows the DSC analysis of the PVP nanocomposite fibers as a function of MWCNT concentrations. PVP is mostly an amorphous polymer that can undergo a transition phase when heated or cooled at a specific temperature, known as the glass transition temperature $\left(T_{g}\right)$, where the polymer is in partly a rubbery and partly a glassy phase. It is related to the segment movement/vibration of the polymer chains. $T_{g}$ is a main function of molecular architecture, monomer units, presence of impurities, nanoscale inclusions, crystallinity,

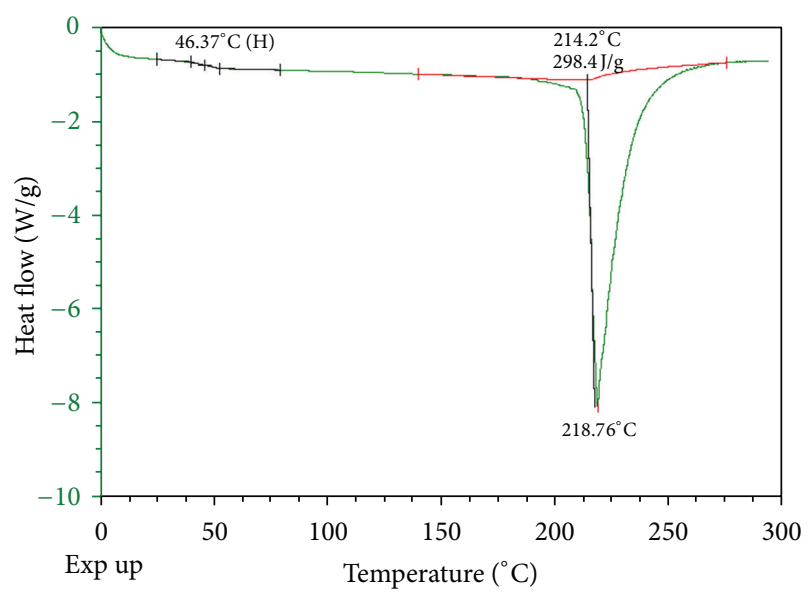

(a)

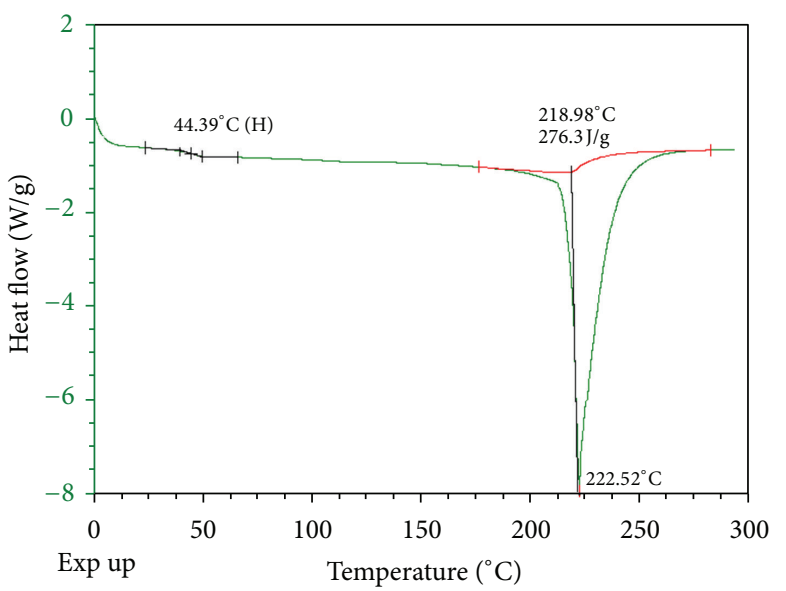

(b)

FIGURE 8: DCS analysis of PVP nanocomposite fibers associated with (a) $0 \mathrm{wt} \%$ and (b) $4 \mathrm{wt} \%$ of MWCNTs.

molecular weight, and the rate of temperature changes [2427]. This transition temperature has been extensively studied for many polymers since it is one of the most fundamental features of polymers. In general, the nanoscale inclusions directly affect the $T_{g}$ of the polymeric nanocomposites, and the interaction of the polymer chains with the surface of the inclusion can significantly alter the chain kinetics of the region that is surrounded by the nanoscale inclusions.

A recent study has shown that nanoscale inclusions in polymers had no significant effects on the glass transition temperature of polymers and polymeric nanocomposites. However, no coherent conclusion has been drawn about the effect of nanoscale inclusions on the $T_{g}$. Strong interactions and possible covalent bonding between nanotubes and polymer matrix most likely result in an increase in $T_{g}$ [24]. Our study also showed that there was no significant effect on the $T_{g}$ (from $46.4^{\circ} \mathrm{C}$ to $44.4^{\circ} \mathrm{C}$ at $4 \mathrm{wt} \%$ ) of PVP fibers incorporated with MWCNT inclusions (Figure 8). This may be attributed to the lack of strong covalent bonds between the MWCNTs and the polymer matrix. The only considerable change we found was the heat of the fusion. For example, the 
heat of the fusion decreased from $298.4 \mathrm{~J} / \mathrm{g}$ to $276.3 \mathrm{~J} / \mathrm{g}$ when the inclusion concentration increased from 0 to $4 \mathrm{wt} \%$.

\section{Conclusions}

In the present study, the effects of external loads and temperatures on the resistance of electrospun polyvinylpyrrolidone nanocomposite fibers were investigated in detail. Those factors that influence the resistance are mainly porosity, charge carrier concentration, and polarizability of inclusions in the polymer matrix. The addition of multiwall carbon nanotubes most likely increased the charge carrier concentration and polarizability and reduced the porosity of the electrospun fiber films under the load. In the load versus temperature experiment, the electrical resistance decreases because the polymer molecules undergo a transition phase in the temperature range of $37^{\circ} \mathrm{C}$ to $60^{\circ} \mathrm{C}$. This transition phase was confirmed by differential scanning calorimetry studies. No significant effect was observed on the glass transition temperature property of the PVP nanocomposite fibers in the presence of MWCNTs, which may suggest that no significant covalent bonds existed between the MWCNTs and the polymer matrix.

\section{Acknowledgment}

The authors would like to acknowledge the Wichita State University for the financial and technical support of this work.

\section{References}

[1] A. Stoiljkovic, M. Ishaque, U. Justus et al., "Preparation of waterstable submicron fibers from aqueous latex dispersion of waterinsoluble polymers by electrospinning," Polymer, vol. 48, no. 14, pp. 3974-3981, 2007.

[2] Z. M. Huang, Y. Z. Zhang, M. Kotaki, and S. Ramakrishna, "A review on polymer nanofibers by electrospinning and their applications in nanocomposites," Composites Science and Technology, vol. 63, no. 15, pp. 2223-2253, 2003.

[3] P. R. Kumar, N. Khan, S. Vivekanandhan, N. Satyanarayana, A. K. Mohanty, and M. Misra, "Nanofibers: effective generation by electrospinning and their applications," Journal of Nanoscience and Nanotechnology, vol. 12, no. 1, pp. 1-25, 2012.

[4] W. S. Khan, Fabrication and characterization of polyvinylpyrrolidone and polyacrylonitrile electrospun nanocomposite fibers [Ph.D. thesis], Wichita State University, Wichita, Kan, USA, 2010.

[5] P. P. llich, Selected Problems in Physical Chemistry-Strategies and Interpretations, Springer, New York, NY, USA, 2010.

[6] A. K. Galwey and M. E. Brown, "Application of the arrhenius equation to solid state kinetics: can this be justified?" Thermochimica Acta, vol. 386, no. 1, pp. 91-98, 2002.

[7] J. J. O'Dwyer, The Theory of Electrical Conduction and Breakdown in Solid Dielectrics, Clarendon Press, Oxford, UK, 1973.

[8] B. Sundaray, Preparation and characterization of electrospun fibers of carbon nanotube-polymer Nanocomposite [Ph.D. thesis], Department of Physic, Indian Institute of Technology, Madras, India, 2006.
[9] X. Zhang, Y. Li, G. Lv, Y. Zuo, and Y. Mu, "Thermal and crystallization studies of nano-hydroxyapatite reinforced polyamide 66 biocomposites," Polymer Degradation and Stability, vol. 91, no. 5, pp. 1202-1207, 2006.

[10] X. Chen, S. Wei, C. Gunesoglu et al., "Electrospun magnetic fibrillar polystyrene nanocomposites reinforced with nickel nanoparticles," Macromolecular Chemistry and Physics, vol. 211, no. 16, pp. 1775-1783, 2010.

[11] J. Zhu, S. Wei, R. Patil et al., "Ionic liquid assisted electrospinning of quantum dots/elastomer composite nanofibers," Polymer, vol. 52, no. 9, pp. 1954-1962, 2011.

[12] D. Zhang, A. B. Karki, D. Rutman et al., "Electrospun polyacrylonitrile nanocomposite fibers reinforced with $\mathrm{Fe} 3 \mathrm{O} 4$ nanoparticles: fabrication and property analysis," Polymer, vol. 50, no. 17, pp. 4189-4198, 2009.

[13] J. Zhu, S. Wei, D. Rutman, N. Haldolaarachchige, D. P. Young, and Z. Guo, "Magnetic polyacrylonitrile-Fe $\mathrm{FeO}$ nanocomposite fibers-electrospinning, stabilization and carbonization," Polymer, vol. 52, no. 13, pp. 2947-2955, 2011.

[14] L. Bokobza, "Multiwall carbon nanotubes-filled natural rubber: electrical and Mechanical properties," Express Polymer Letters, vol. 6, no. 3, pp. 213-223, 2012.

[15] W. S. Khan, R. Asmatulu, and M. B. Yildirim, "Acoustical properties of electrospun fibers for aircraft interior noise reduction," Journal of Aerospace Engineering, vol. 25, no. 3, pp. 376-382, 2012.

[16] A. Szentes, C. S. Varga, G. Horvath et al., "Electrical resistivity and thermal properties of compatibilized multi-walled crabon nanotube/Polypropylene composites," EXpress Polymer Letters, vol. 6, no. 6, pp. 494-502, 2012.

[17] J. Zhu, S. Wei, J. Ryu, and Z. Guo, "Strain sensing elastomer/carbon nanofibers 'Metacomposites"' Journal of Physical Chemistry C, vol. 115, no. 27, pp. 13215-13222, 2011.

[18] N. F. Mott and E. A. Davis, Electronic Process on Non-Crystalline Mateials, Oxford University Press, New York, NY, USA, 2nd edition, 1979.

[19] L. Wang, L. Zhang, and M. Tian, "Improved polyvinylpyrrolidone (PVP)/graphite nanocomposites by solution compounding and spray drying," Polymers for Advanced Technologies, vol. 23, no. 3, pp. 652-659, 2012.

[20] F. H. Gojny, M. H. G. Wichmann, B. Fiedler et al., "Evaluation and identification of electrical and thermal conduction mechanisms in carbon nanotube/epoxy composites," Polymer, vol. 47, no. 6, pp. 2036-2045, 2006.

[21] B. Sundaray, V. J. Babu, V. Subramanian, and S. T. Natarajan, "Preparation and characterization of electrospun fibers of Poly(Methyl Methacrylate) single walled carbon notube composites," Journal of Engineered Fibers and Fabrics, vol. 3, no. 4, pp. 39-45, 2008.

[22] L. Bokobza, "Multiwall carbon nanotube-filled natural rubber: electrical and Mechanical Properties," Express Polymer Letters, vol. 6, no. 3, pp. 213-223, 2012.

[23] N. Norkhairunnisa, A. Azizan, M. Mariatti, H. Ismail, and L. C. Sim, "Thermal stability and electrical behavior of polydimethylsiloxane nanocomposites with carbon nanotubes and carbon black fillers," Journal of Composite Materials, vol. 46, no. 8, pp. 903-910, 2011.

[24] B. Qiao, X. Ding, X. Hou, and S. Wu, "Study on the electrospun CNTs/polyacrylonitrile based nanofiber composites," Journal of Nanomaterials, vol. 2011, Article ID 839462, 7 pages, 2011. 
[25] P. Gupta, R. Asmatulu, R. Claus, and G. Wilkes, "Superparamagnetic flexible substrates based on submicron electrospun Estane fibers containing MnZnFe-Ni nanoparticles," Journal of Applied Polymer Science, vol. 100, no. 6, pp. 4935-4942, 2006.

[26] W. S. Khan, R. Asmatulu, Y. H. Lin, Y. Y. Chen, and J. Ho, "Electrospun polyvinylpyrrolidone-based nanocomposite fibers containing $\left(\mathrm{Ni}_{0.6} \mathrm{Zn}_{0.4}\right) \mathrm{Fe}_{2} \mathrm{O}_{4}$," Journal of Nanotechnology, vol. 2012, Article ID 138438, 5 pages, 2012.

[27] R. Asmatulu, B. Zhang, and N. Nuraje, "A ferrofluid guided system for the rapid separation of the non-magnetic particles in a microfluidic device," Journal of Nanoscience and Nanotechnology, vol. 10, no. 10, pp. 6383-6387, 2010.

[28] M. Naebe, T. Lin, M. P. Staiger, L. Dai, and X. Wang, "Electrospun single-walled carbon nanotube/polyvinyl alcohol composite nanofibers: structure-property relationships," Nanotechnology, vol. 19, no. 30, Article ID 305702, 2008.

[29] W. Edward and W. D, Morphological and mechanical properties of carbon nanotubes/polymer composites via melt compounding [M.S. thesis], North Carolina State University, Raleigh, NC, USA, 2005 

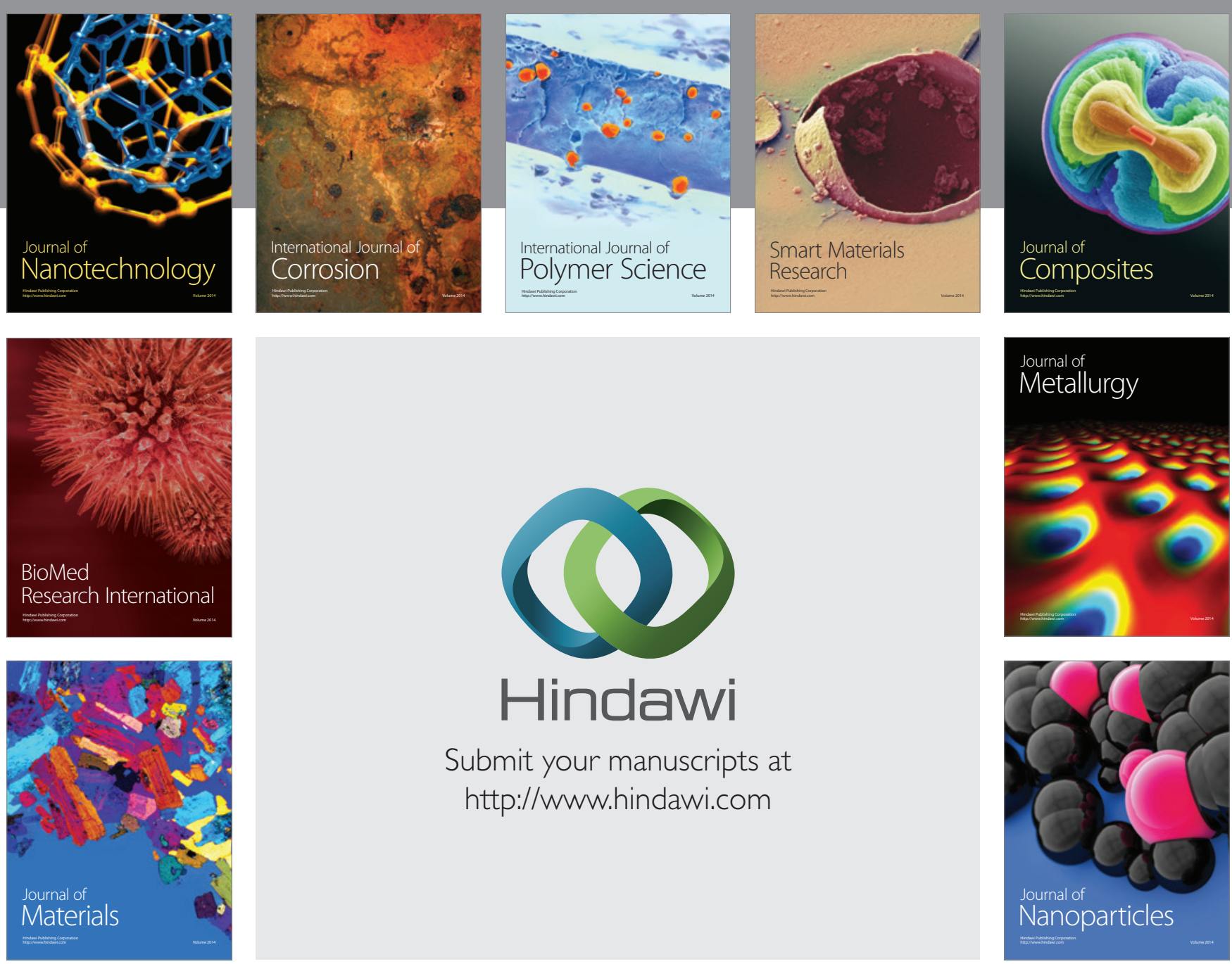

Submit your manuscripts at http://www.hindawi.com
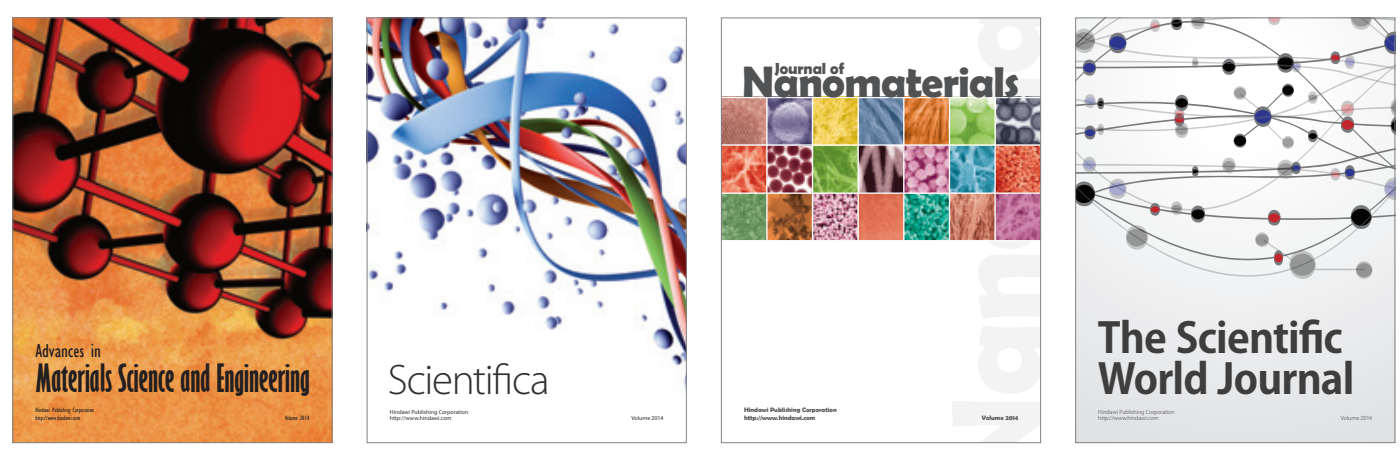

\section{The Scientific World Journal}
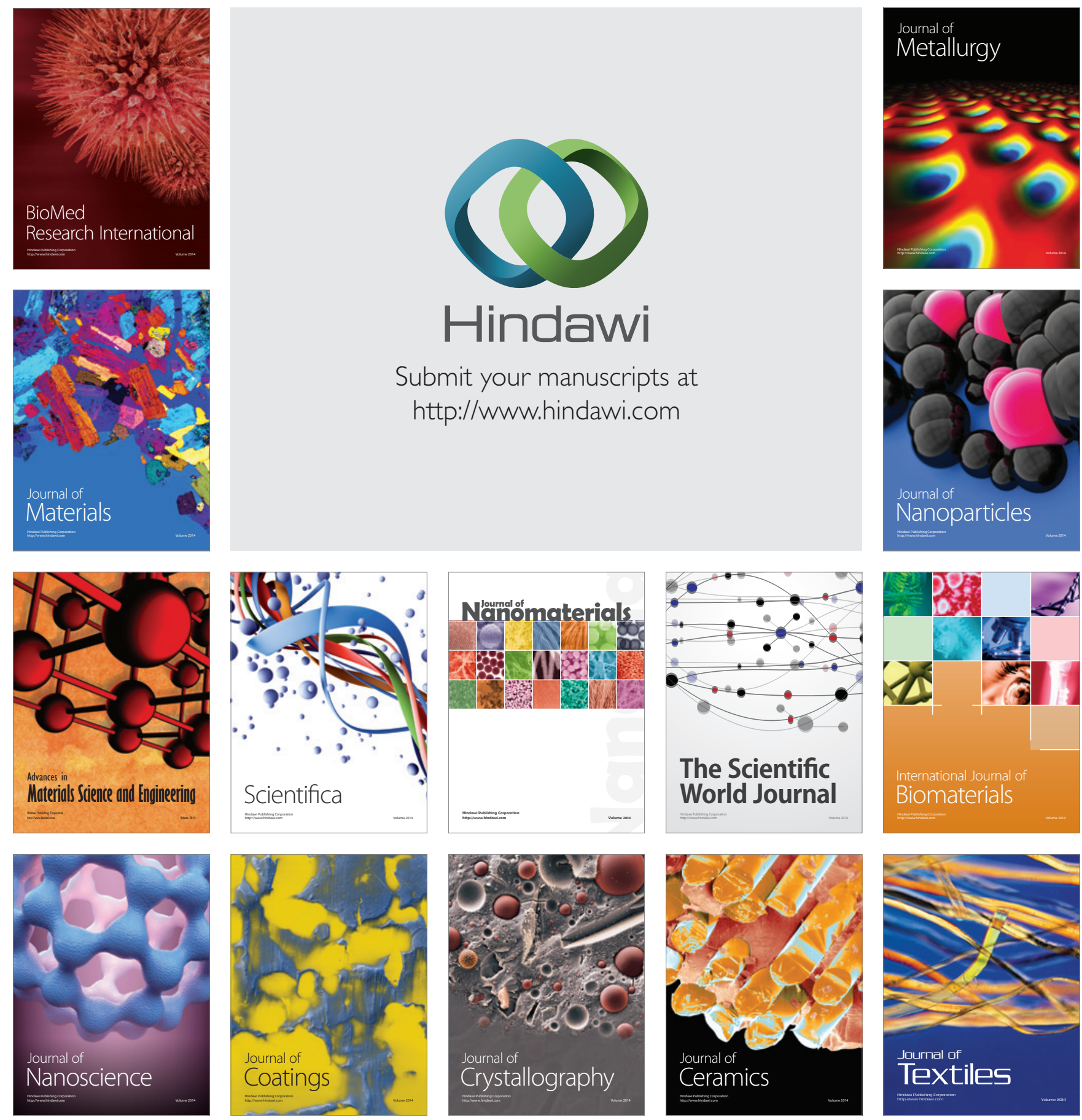